\title{
Itch Matrixes
}

\begin{abstract}
Peyman Najafi ${ }^{1,2}$, Laurent Misery ${ }^{1,3 *}$, Jean-Luc Carré ${ }^{1}$, Douraied Ben Salem ${ }^{4,5}$ and Olivier Dufor ${ }^{1,6}$

${ }^{1}$ Univ Brest, LIEN, Brest, France, ${ }^{2}$ Paris-Saclay Institute of Neuroscience, Centre National de la Recherche Scientifique/ Université Paris-Saclay, Gif-sur-Yvette, France, ${ }^{3}$ University Hospital of Brest, Department of Dermatology, Brest, France, ${ }^{4}$ Univ Brest, LATIM, INSERM UMR, Brest, France, ${ }^{5}$ University Hospital of Brest, Department of Radiology, Brest, France, ${ }^{6}$ L@b/SEN Yncréa Ouest, ISEN, Brest, France
\end{abstract}

Keywords: itch, matrix, pain, brain, imaging

\section{INTRODUCTION}

In a recent meta-analysis from our group based on a systematic review we have identified brain regions reported to be responsible for central mechanisms of itch processing (1). We also have discussed the central mechanisms of itch proceeding in the brain more in depth in a review paper (2). The research papers that have studied central mechanism of itch are presented in Table $\mathbf{1}$ while their results are presented in Table 2. Here in this paper, we are exploring a new idea in which we categorize the itch matrix in the brain into three matrixes that each of them is contributing to a specific aspect of itch perception. This conceptualizes the processing of itch signals into different

OPEN ACCESS

Edited by:

Sonja Ständer,

University Hospital Münster, Germany

Reviewed by:

Svetlana Bobko,

Moscow Scientific and Practical

Center for Dermatovenerology and

Cosmetology, Russia

Jesper Elberling,

Gentofte Hospital, Denmark

*Correspondence:

Laurent Misery

laurent.misery@chu-brest.fr

Specialty section:

This article was submitted to Dermatology,

a section of the journal

Frontiers in Medicine

Received: 02 December 2020 Accepted: 25 January 2021

Published: 24 February 2021

Citation:

Najafi P, Misery L, Carré J-L, Ben Salem D and Dufor O (2021) Itch Matrixes. Front. Med. 8:636904. doi: 10.3389/fmed.2021.636904 itch matrices could be useful in order to model different aspects of itch. For example, it is possible, that an overactivity in second matrix cause a higher susceptivity to contagious itch.

Unlike the visual system pain and itch can evoke multitude of regions in the brain, which we call pain matrix and itch matrix respectively. Recent studies have proposed that the pain matrix can be categorized into three different pain matrixes $(40,41)$ : one contributing to perception and the location of pain; another matrix responsible for the affective aspect of the pain; and a third involving decoding the cognitive aspect of pain. In the same manner, we guardedly propose that the itch processing network can be broken down into three main matrixes although many data are still lacking. These three matrixes have been presented in Figure 1.

\section{FIRST ITCH MATRIX}

The first itch matrix includes but is not restricted to the primary sensorimotor cortex, the parietal/central operculum, and the posterior insular cortex (Figure 2A).

Among these three regions the primary sensorimotor cortex is involved in the encoding of the recognition, localization, and intensity of painful stimuli (42). In pain studies, activation in this region bears a linear relationship with pain intensity (43-47). In a positron emission tomography (PET) study by Drzezga et al. (5) the authors reported that SI activity, is positively correlated with itch intensity. Six years after Drzezga, in 2007, Mochizuki et al. added the secondary somatosensory cortex (SII) demonstrating an increase of activity in this region after itch induction with histamine (10). The increase was statistically not different than the proven one observed in the painful condition (pain vs. itch) but did not reach a statistically corrected threshold when comparing itch against no itch.

In another study which includes both $\mathrm{AD}$ patients and healthy controls, itch was found to activate the post-central gyrus in the right hemisphere (12). This study together with Drzezga study in 2001 are reported in the meta-analysis on Itch from Lee et al. (48). Out of 56 regions listed in the parietal cortex (31 Left and 25 Right) from 18 studies (Table 1). Brain activity upon itch stimulation, 
in (48), left SI appears to be activated eight times against two only in the right hemisphere. On the contrary, right SII is reported five times against two only in the left hemisphere. The other regions mentioned $(n=39)$ are in both left and right parietal cortices sometime very near to the SI/SII regions (i.e., SMG, SPL, IPL, anterior parietal cortex).

In the meta-analysis from Roberts et al. (49), the authors suggest the possibility of a specificity of these regions for the itching process as they appear to be better activated by itching than by pain. Interestingly, they also group these regions with the central operculum. In a recent meta-analysis of our group (1), SI/SII region was not clearly identified but we discussed this point regarding the diversity of studies we included. Our results on correlations with itch intensity also showed two important clusters in bilateral insular cortices (5068 voxels right 4589 voxels left) that spread to a great extent on the postcentral gyri.

The co-activation of the central operculum together with SI/SII cortex is widely reported in itch literature both in healthy subjects and patients. Indeed, central operculum corresponding to the junction of pre- and post-central gyri accompanied with the region located laterally to the posterior convolution of the insula is often confounded with insula itself or even SI. In the regions abbreviated OPC, also named rolandic operculum elsewhere, itch intensity was also correlated with PET signal both in healthy subjects and $\mathrm{AD}$ patients $(4,33)$.

Finally, we propose that the insular cortex, and especially its posterior portion, takes part into this first matrix. As a common point between these regions, their gradual response with itch intensity seems important to highlight. In Leknes et al. bilateral insular and left posterior insular activity (BOLD) is correlated with histamine-induced itch intensity (9). Following Craig (50, 51), Mochizuki et al. postulate that the posterior part of insula plays a different role than its anterior part $(52,53)$. A distinction that can also find its basis on cytoarchitectural composition of these structures and their connectives with other brain areas $(50,54)$.

Despite weak evidences in itch literature, other evidences can help to understand the insula role in processing the sensations which are common to itch and pain. Mazzola et al. explain that the two thirds of posterior insula submitted to low electrical stimulation (SEEG) directly translate these stimulations as pain sensations (55). Another study from Frot et al. showed that once pain feeling is reached, the posterior insular

\footnotetext{
Abbreviations: ACC, Anterior Cingulate Cortex; AD, Atopic Dermatitis; aIC, Anterior part of the Insular Cortex; aMCC, Anterior part of the Middle Cingulate Cortex; BOLD, Blood Oxygenation Level Dependent; dACC, Dorsal part of the Anterior Cingulate Cortex; DLPFC, Dorso-Lateral Pre-Frontal Cortex; IPL, Inferior Parietal Lobule; OPC, Operculum Central; PAG, Peri-Aqueductal Gray matter; PCC, Posterior Cingulate Cortex; PET, Positron Emission Tomography; pIC, Posterior Insular Cortex; pMCC, Posterior part of the Middle Cingulate Cortex; SI, Primary Somatosensory cortex; SII, Secondary Somatosensory cortex; SEEG, Stereo-Electro-Encephalo-Graphy; SMG, Supra-Marginal Gyrus; SPL, Superior Parietal Lobule.
}

cortex activity still correlates with noxious thermal stimulation intensity (47).

In summary, all these regions encode the feeling of itchy sensation and are somewhat translating its intensity level as well as their location following a somatotopic representation. When compared to Xiang et al. study (41), this first matrix includes all already reported regions for pain. However, studies reporting activities in those regions only for itch are rare and some studies need to be carefully interpreted given approximations inherent to main peak reporting. Effectively, secondary peaks of wide clusters or percentage of anatomical regions covered by these clusters are most often not indicated. As an example, the absence of parietal operculum in Roberts et al. study (49) needs to be put in perspective. Indeed, the point that the contrast pain-itch shows an increased activity in the parietal operculum does not mean that this region is silent in itch. Moreover, in the same study, the opposite contrast itch-pain, which reveals an implication of both right supramarginal gyrus and central operculum, could have led us to add more parietal areas to this first matrix.

So far, we have dealt with the membership of each of these brain regions in the matrix separately. However, interesting arguments reside in the fact that new pathological conditions can appear when these regions grouped and malfunction together. Hence, some studies reported that SI/SII together with the insular cortex participate in creating the allodynia phenomenon (56-59). Consecutively, these regions once activated lead to an ignition of the pain network inducing activity in the PAG, the prefrontal cortex, the thalamus, the amygdala, the ACC and many other regions within the pain network. Allodynia has repercussions on the way normal brain areas react to tactile stimuli and authors do not only consider the condition through the scope of pain matrix. Many brain areas are those involved in tactile or thermal sensitivity and this allows more faithful comparison with itch perception. The difficulty with allodynia is that even when it is spontaneous, painful sensation is quickly reached and its intensity then depends on other brain region listed above.

To illustrate this phenomenon, we adduce together both Ducreux et al. study (60) and an article from Geuter et al. (61) about predictive coding. In Ducreux et al. authors demonstrated with noxious and non-noxious cold stimulation $\left(4^{\circ}\right.$ and $\left.22^{\circ} \mathrm{C}\right)$ that while non-noxious cold in control subjects activates SII and the insular cortex (mostly its anterior part), the same nonnoxious stimulation did activates SII and mid-posterior insula in allodynic patients together with other regions of the pain network (60). In Geuter et al. work, the authors used the predictive coding theory of brain functioning to demonstrate a difference within the anterior and the posterior part of the insula. While the anterior part would be dedicated to pain feelings as a prediction error on perceived sensations, the posterior part only responds to pain intensity with no comparisons to any predicted sensation (61). We propose that in Ducreux et al. even if the feeling is non-noxious in control subjects, it remains unpredictable and then activates the anterior part of the insula. However, allodynic patients are prepared to feel painful 
TABLE 1 | Papers and methods which have been used in order to study central mechanism of itch.

\begin{tabular}{|c|c|c|c|c|c|c|c|c|}
\hline$\#$ & References & Scanner & $\begin{array}{l}\text { Neuroimaging } \\
\text { analysis }\end{array}$ & $\begin{array}{l}\text { Itch } \\
\text { induction }\end{array}$ & Itch stimulus & $\begin{array}{c}\text { Number } \\
\text { of } \\
\text { subjects }\end{array}$ & Pathology & Comments \\
\hline 2 & Darsow et al. (4) & PET & Subtraction & Skin prick & Histamine & 6 & Healthy & \\
\hline 3 & Darsow et al. (4) & PET & Correlation & Skin prick & Histamine & 6 & Healthy & \\
\hline 5 & Mochizuki et al. (6) & PET & Subtraction & lontophoresis & Histamine & 15 & Healthy & \\
\hline 6 & Walter et al. (7) & $\mathrm{fMRI}$ & Correlation & Skin prick & Histamine & 6 & Healthy & \\
\hline 7 & Herde et al. (8) & $\mathrm{fMRI}$ & Subtraction & $\begin{array}{l}\text { Intracutaneous } \\
\text { microdialysis }\end{array}$ & Histamine & 10 & Healthy & \\
\hline 8 & Leknes et al. (9) & $\mathrm{fMRI}$ & Correlation & Skin prick & Histamine & 8 & Healthy & \\
\hline 9 & Leknes et al. (9) & fMRI & Correlation & & Allergan & 8 & Atopic cohort & \\
\hline 13 & Valet et al. (11) & $\mathrm{fMRI}$ & Subtraction & Skin prick & Histamine & 12 & Healthy & $\begin{array}{l}\text { Temperature } \\
\text { modeling }\end{array}$ \\
\hline 14 & Schneider et al. (12) & PET & Subtraction & lontophoresis & Histamine & 6 & Healthy & \\
\hline 15 & Schneider et al. (12) & PET & Subtraction & Iontophoresis & Histamine & 8 & $\begin{array}{l}\text { Atopic } \\
\text { dermatitis }\end{array}$ & \\
\hline 16 & Schneider et al. (12) & PET & Subtraction & lontophoresis & Histamine & 8 & $\begin{array}{l}\text { Healthy }<> \\
A D\end{array}$ & \\
\hline 17 & Yosipovitch et al. (13) & $\mathrm{fMRI}$ & Subtraction & Scratching & & 13 & Healthy & \\
\hline 18 & Ishiuji et al. (14) & $\mathrm{fMRI}$ & ASL & Iontophoresis & Histamine & 8 & $\begin{array}{l}\text { Atopic } \\
\text { dermatitis }\end{array}$ & \\
\hline 19 & Ishiuji et al. (14) & $\mathrm{fMRI}$ & ASL & lontophoresis & Histamine & 7 & Healthy & \\
\hline 25 & Pfab et al. (17) & fMRI & Subtraction & $\begin{array}{l}\text { Skin prick non } \\
\text { lesion skin }\end{array}$ & Histamine & 13 & $\begin{array}{l}\text { Atopic } \\
\text { dermatitis }\end{array}$ & $\begin{array}{l}\text { Thermal } \\
\text { modulation }\end{array}$ \\
\hline 26 & Pfab et al. (17) & fMRI & Subtraction & $\begin{array}{l}\text { Skin prick } \\
\text { lesion skin }\end{array}$ & Histamine & 13 & $\begin{array}{l}\text { Atopic } \\
\text { dermatitis }\end{array}$ & $\begin{array}{l}\text { Thermal } \\
\text { modulation }\end{array}$ \\
\hline 27 & Bergeret et al. (18) & PET & Subtraction & lontophoresis & Histamine & 28 & Healthy & \\
\hline 28 & Bergeret et al. (18) & PET & Correlation & lontophoresis & Histamine & 29 & Healthy & Itch sensation \\
\hline 29 & Holle et al. (19) & $\mathrm{fMRI}$ & Subtraction & $\begin{array}{l}\text { Audiovisual } \\
\text { itch }\end{array}$ & & 18 & Healthy & \\
\hline 30 & Holle et al. (19) & $\mathrm{fMRI}$ & Correlation & $\begin{array}{l}\text { Audiovisual } \\
\text { itch }\end{array}$ & & 19 & Healthy & \\
\hline 31 & Kleyn et al. (20) & fMRI & Subtraction & Skin prick & Histamine & 16 & Healthy & \\
\hline 32 & Kleyn et al. (20) & $\mathrm{fMRI}$ & Correlation & Skin prick & Histamine & 16 & Healthy & \\
\hline 33 & Papoiu et al. (21) & $\mathrm{fMRI}$ & ASL & lontophoresis & Histamine & 15 & Healthy & \\
\hline 34 & Papoiu et al. (21) & $\mathrm{fMRI}$ & ASL & $\begin{array}{l}\text { Spicules } \\
\text { rubbing }\end{array}$ & Cowhage & 15 & Healthy & \\
\hline
\end{tabular}


TABLE 1 | Continued

\begin{tabular}{|c|c|c|c|c|c|c|c|c|}
\hline$\#$ & References & Scanner & $\begin{array}{l}\text { Neuroimaging } \\
\text { analysis }\end{array}$ & $\begin{array}{l}\text { Itch } \\
\text { induction }\end{array}$ & Itch stimulus & $\begin{array}{c}\text { Number } \\
\text { of } \\
\text { subjects }\end{array}$ & Pathology & Comments \\
\hline 36 & Papoiu et al. (21) & fMRI & Subtraction & $\begin{array}{l}\text { Audiovisual } \\
\text { pain }\end{array}$ & & 18 & Healthy & \\
\hline 39 & Papoiu et al. (22) & fMRI & $\begin{array}{l}\text { ASL- } \\
\text { correlation }\end{array}$ & Scratching & & 14 & Healthy & $\begin{array}{l}\text { Correlated with } \\
\text { itch relief }\end{array}$ \\
\hline 40 & Stumpf et al. (23) & fMRI & Subtraction & Microdialysis & Histamine & 33 & Healthy & Female $>$ Males \\
\hline 43 & Napadow et al. (24) & fMRI & Subtraction & Skin prick & $\begin{array}{l}\text { Allergen- } \\
\text { induced }\end{array}$ & 14 & $\begin{array}{l}\text { Atopic } \\
\text { dermatitis }\end{array}$ & $\begin{array}{l}\text { Temperature } \\
\text { modeling and } \\
\text { acupuncture } \\
\text { intervention }\end{array}$ \\
\hline 44 & Desbordes et al. (25) & fMRI & Connectivity & Skin prick & $\begin{array}{l}\text { Allergen- } \\
\text { induced }\end{array}$ & 14 & $\begin{array}{l}\text { Atopic } \\
\text { dermatitis }\end{array}$ & $\begin{array}{l}\text { Right premotor } \\
\text { as seed }\end{array}$ \\
\hline 45 & Desbordes et al. (25) & fMRI & Connectivity & Skin prick & $\begin{array}{l}\text { Allergen- } \\
\text { induced }\end{array}$ & 14 & $\begin{array}{l}\text { Atopic } \\
\text { dermatitis }\end{array}$ & $\begin{array}{l}\text { Right insula as } \\
\text { seed }\end{array}$ \\
\hline 46 & Desbordes et al. (25) & fMRI & Connectivity & Skin prick & $\begin{array}{l}\text { Allergen- } \\
\text { induced }\end{array}$ & 14 & $\begin{array}{l}\text { Atopic } \\
\text { dermatitis }\end{array}$ & $\begin{array}{l}\text { Right putamen } \\
\text { as seed }\end{array}$ \\
\hline 47 & Desbordes et al. (25) & fMRI & Connectivity & Skin prick & $\begin{array}{l}\text { Allergen- } \\
\text { induced }\end{array}$ & 14 & $\begin{array}{l}\text { Atopic } \\
\text { dermatitis }\end{array}$ & $\begin{array}{l}\text { Left superior } \\
\text { parietal lobule } \\
\text { as seed }\end{array}$ \\
\hline 51 & Mochizuki et al. (26) & fMRI & Subtraction & $\begin{array}{l}\text { Electrically } \\
\text { induced itch }\end{array}$ & & 16 & Healthy & \\
\hline 52 & Mochizuki et al. (26) & $\mathrm{fMRI}$ & Subtraction & $\begin{array}{l}\text { Electrically } \\
\text { induced itch }\end{array}$ & $\begin{array}{l}\text { Passive } \\
\text { scratching }\end{array}$ & 16 & Healthy & Scratching itch \\
\hline 53 & Mochizuki et al. (26) & fMRI & Subtraction & $\begin{array}{l}\text { Electrically } \\
\text { induced itch }\end{array}$ & $\begin{array}{l}\text { Passive } \\
\text { scratching }\end{array}$ & 16 & Healthy & $\begin{array}{l}\text { Scratching } \\
\text { itch> } \\
\text { scratching } \\
\text { another region }\end{array}$ \\
\hline 54 & Mochizuki et al. (26) & fMRI & Subtraction & $\begin{array}{l}\text { Electrically } \\
\text { induced itch }\end{array}$ & $\begin{array}{l}\text { Passive } \\
\text { scratching }\end{array}$ & 16 & Healthy & $\begin{array}{l}\text { Deactivation } \\
\text { scratching itch } \\
\text { region }\end{array}$ \\
\hline 55 & Mochizuki et al. (26) & fMRI & Subtraction & $\begin{array}{l}\text { Electrically } \\
\text { induced itch }\end{array}$ & $\begin{array}{l}\text { Passive } \\
\text { scratching }\end{array}$ & 16 & Healthy & $\begin{array}{l}\text { Scratching } \\
\text { another region }\end{array}$ \\
\hline 56 & Papoiu et al. (27) & fMRI & ASL & Iontophoresis & Histamine & 13 & $\begin{array}{l}\text { End-stage } \\
\text { renal disease }\end{array}$ & \\
\hline
\end{tabular}


TABLE 1 | Continued

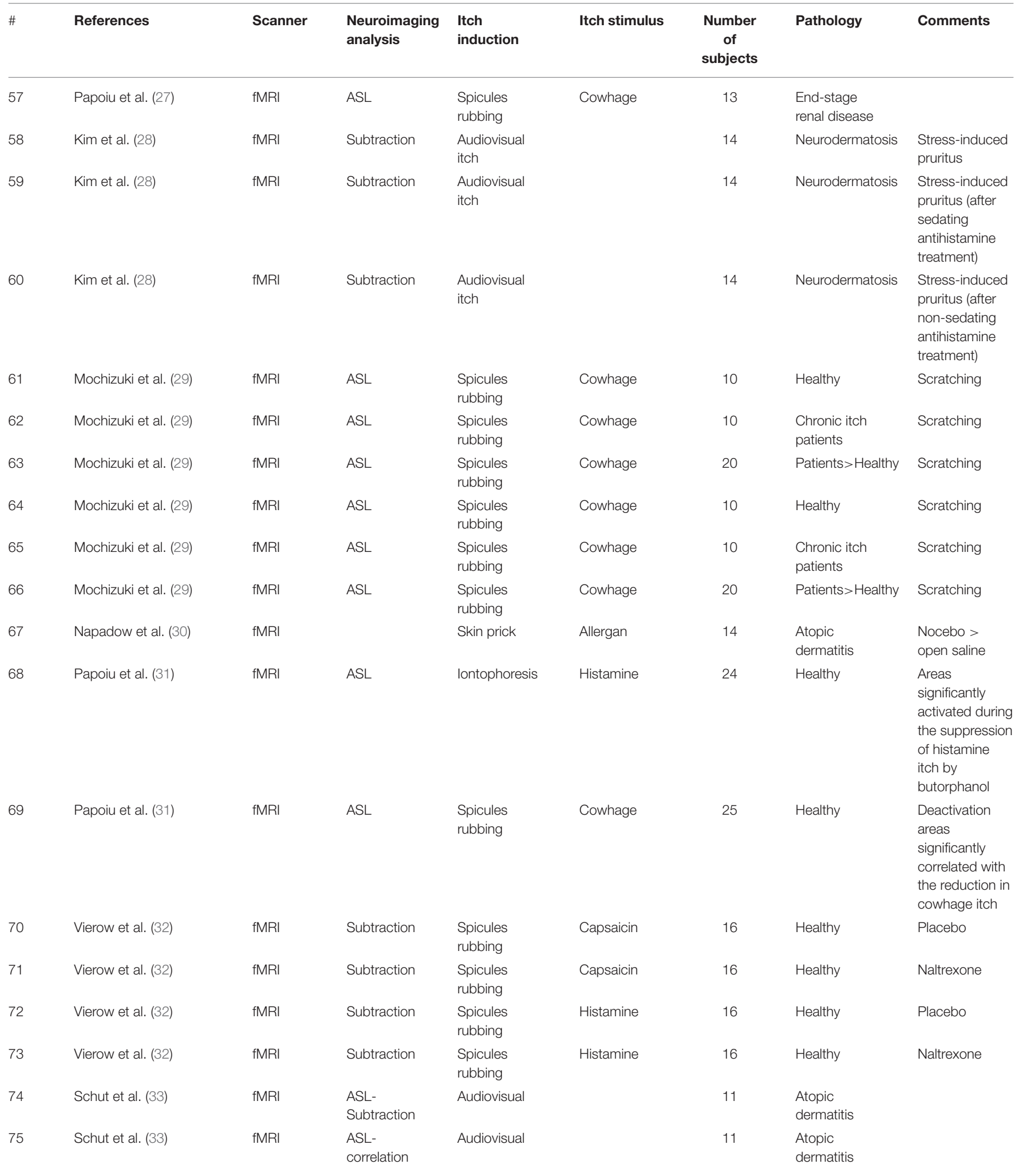


TABLE 1 | Continued

\begin{tabular}{|c|c|c|c|c|c|c|c|c|}
\hline$\#$ & References & Scanner & $\begin{array}{l}\text { Neuroimaging } \\
\text { analysis }\end{array}$ & $\begin{array}{l}\text { Itch } \\
\text { induction }\end{array}$ & Itch stimulus & $\begin{array}{c}\text { Number } \\
\text { of } \\
\text { subjects }\end{array}$ & Pathology & Comments \\
\hline 77 & van de Sand et al. (35) & fMRI & Subtraction & Skin patch & Histamine & 30 & Healthy & $\begin{array}{l}\text { Nocebo } \\
\text { modulation } \\
\text { Itch-nocebo > } \\
\text { itch only } \\
\text { (temperature } \\
\text { modulating) }\end{array}$ \\
\hline 78 & van de Sand et al. (35) & fMRI & $\begin{array}{l}\text { Connectivity } \\
\text { with insula }\end{array}$ & Skin patch & Histamine & 30 & Healthy & $\begin{array}{l}\text { Nocebo } \\
\text { modulation } \\
\text { Itch-nocebo > } \\
\text { itch only } \\
\text { (temperature } \\
\text { modulating) }\end{array}$ \\
\hline 80 & Wang et al. (36) & $\mathrm{fMRI}$ & $\begin{array}{l}\text { Resting } \\
\text { state }\end{array}$ & & & $40+40$ & $\begin{array}{l}\text { Chronic } \\
\text { urticaria } \\
\text { +Healthy }\end{array}$ & $\begin{array}{l}\mathrm{CSU}>\mathrm{HC} \\
\text { (functional } \\
\text { connectivity } \\
\text { with right } \\
\text { ventral striatum) }\end{array}$ \\
\hline 81 & Wang et al. (36) & fMRI & $\begin{array}{l}\text { Resting } \\
\text { state }\end{array}$ & & & $40+40$ & $\begin{array}{l}\text { Chronic } \\
\text { urticaria } \\
+ \text { Healthy }\end{array}$ & $\begin{array}{l}\mathrm{CSU}>\mathrm{HC} \\
\text { (functional } \\
\text { connectivity } \\
\text { with right } \\
\text { putamen) }\end{array}$ \\
\hline 84 & Wang et al. (37) & fMRI & $\begin{array}{l}\text { Resting } \\
\text { state }\end{array}$ & & & $40+40$ & $\begin{array}{l}\text { Chronic } \\
\text { urticaria } \\
\text { +Healthy }\end{array}$ & $\begin{array}{l}\mathrm{CSU}>\mathrm{HC} \\
\text { (functional } \\
\text { connectivity } \\
\text { with } \\
\text { Cerebellum) }\end{array}$ \\
\hline 85 & Wang et al. (37) & $\mathrm{fMRI}$ & $\begin{array}{l}\text { Resting } \\
\text { state }\end{array}$ & & & 40 & $\begin{array}{l}\text { Chronic } \\
\text { urticaria }\end{array}$ & $\begin{array}{l}\text { After } \\
\text { intervention > } \\
\text { Before } \\
\text { intervention } \\
\text { (functional } \\
\text { connectivity } \\
\text { with } \\
\text { Cerebellum) }\end{array}$ \\
\hline
\end{tabular}


TABLE 1 | Continued

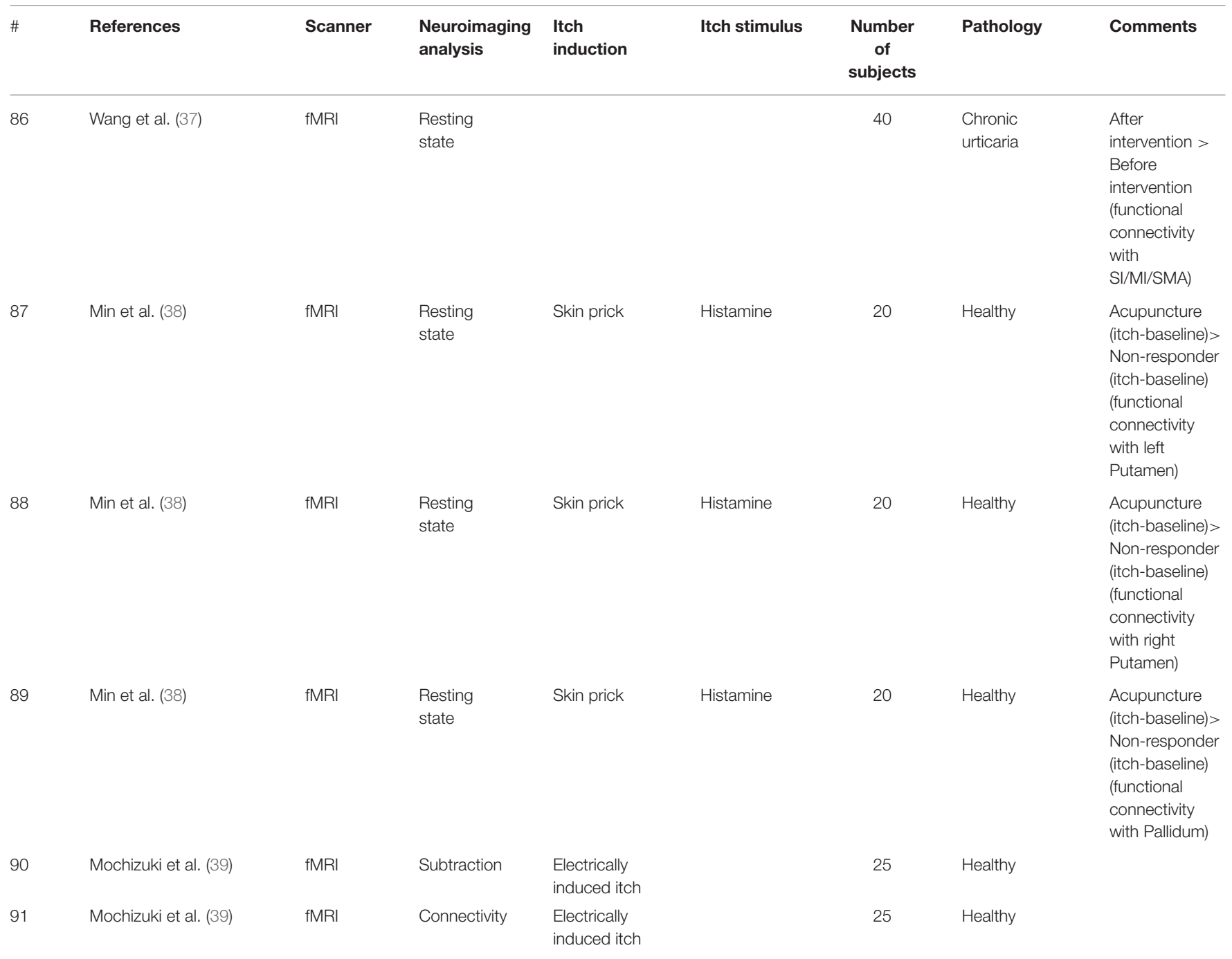

stimulation and then, the anterior part shut down as painful sensation are correctly predicted. Meanwhile, the posterior part of the insula starts to encode its intensity like it was demonstrated by Frot et al. (47) in implanted subjects when stimulation becomes noxious.

\section{SECOND ITCH MATRIX}

The second itch matrix could consist of the ACC, aMCC, aIC, amygdala, striatum and hippocampus (Figure 2B). This network could encode the affective and motivational aspects of itch. Significant activation in the ACC, especially dorsal, extending to the anterior part of the middle cingulate cortex (aMCC), has been linked to the reward network and the positive or negative emotional response (40). Noteworthy, Vogt has reported that the aMCC reflects emotional awareness and fear leading to the questioning of the enrolment of the aMCC to the ACC gross function $(62,63)$. Considering the anterior insula, it is reported to be involved in the awareness of emotions and subjective feelings (50) as well as errors of predictions like mentioned above. Another literature about lesions in the aIC would cause deficits in emotional awareness (e.g., alexithymia) (64). Several studies have reported that activity in the aIC is significantly correlated with the unpleasantness of itch $(8-10,18,21)$. For the hippocampus, it has been also shown that this structure is fully integrated in the itch network $(13,21,22)$. For example, only active scratching can relief activity in ipsi-hippocampal structure (53). The role of hippocampus together with amygdala, dACC and insular cortex are well-documented in Sanders and Akiyama (65). The authors noticed and argued that "amygdala and hippocampus activation appears to go hand-in-hand in most studies of itch, suggesting that the memory of previous itch experiences may be a significant factor in itch-related anxiety." Stratum possibly 
TABLE 2 | Results of the all the papers studied the central mechanism of itch.

\begin{tabular}{|c|c|c|c|c|c|c|c|c|c|c|c|c|c|c|c|c|c|c|c|c|c|c|c|c|c|c|c|c|c|c|c|c|c|}
\hline $\begin{array}{l}\text { Parts of } \\
\text { which matri }\end{array}$ & & 1 & & & & $1 / 2$ & 3 & 2 & 3 & 3 & 3 & & & & 2 & 1 & & 2 & & & 2 & & & & & & & & & 2 & & 2 & \\
\hline Regions & 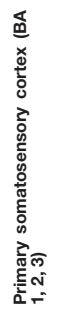 & 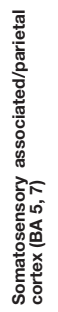 & 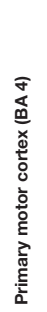 & 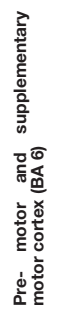 & 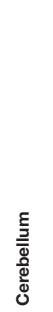 & 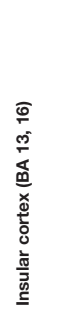 & 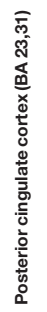 & 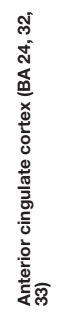 & 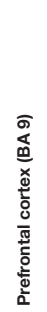 & 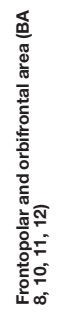 & 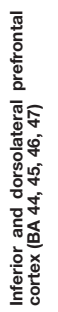 & 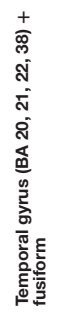 & 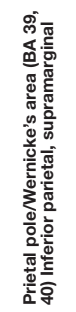 & 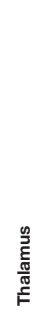 & 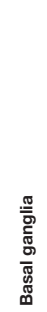 & 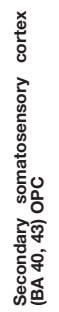 & 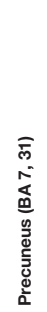 & 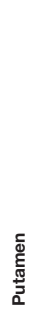 & 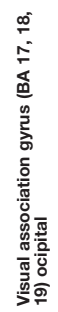 & 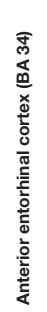 & 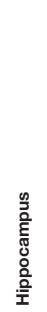 & 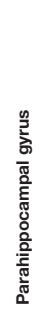 & 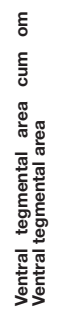 & 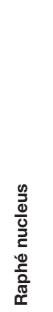 & 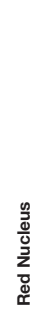 & $\frac{\pi}{2}$ & 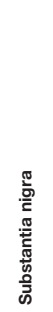 & 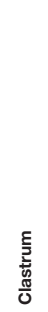 & 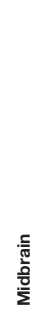 & 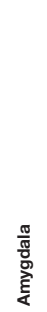 & 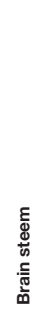 & 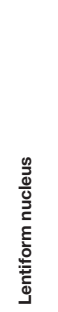 & ذั \\
\hline
\end{tabular}

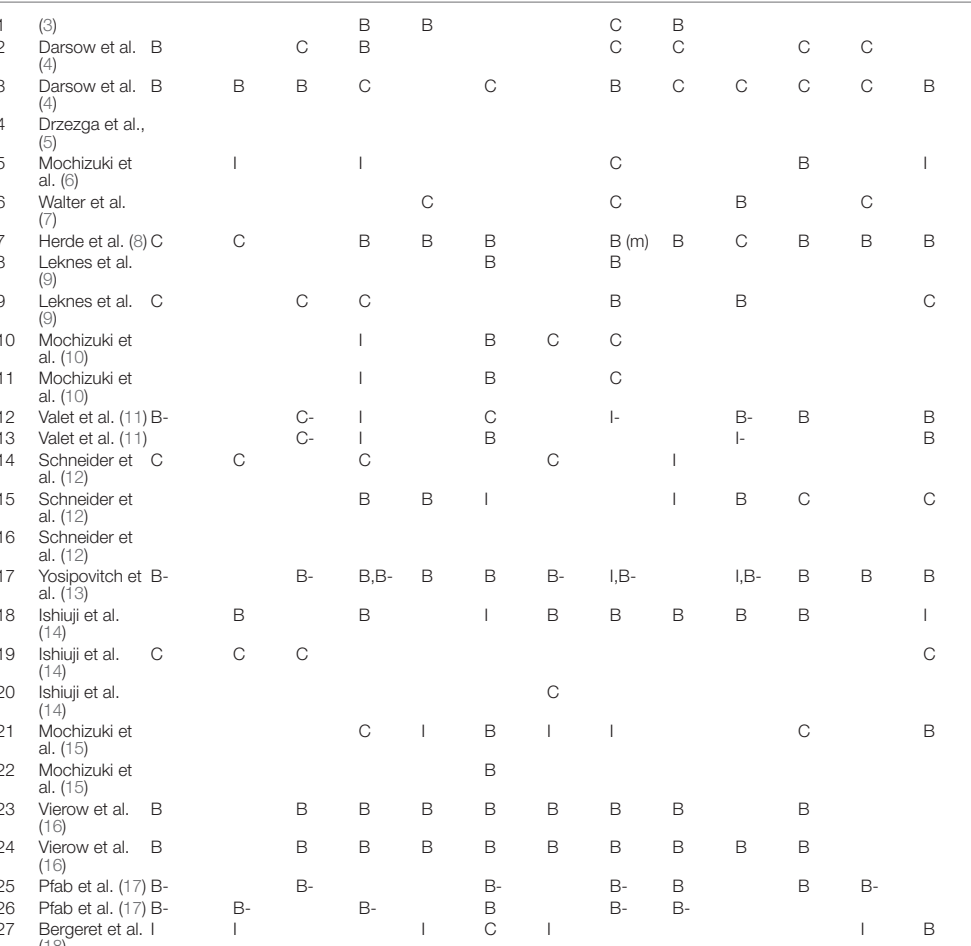




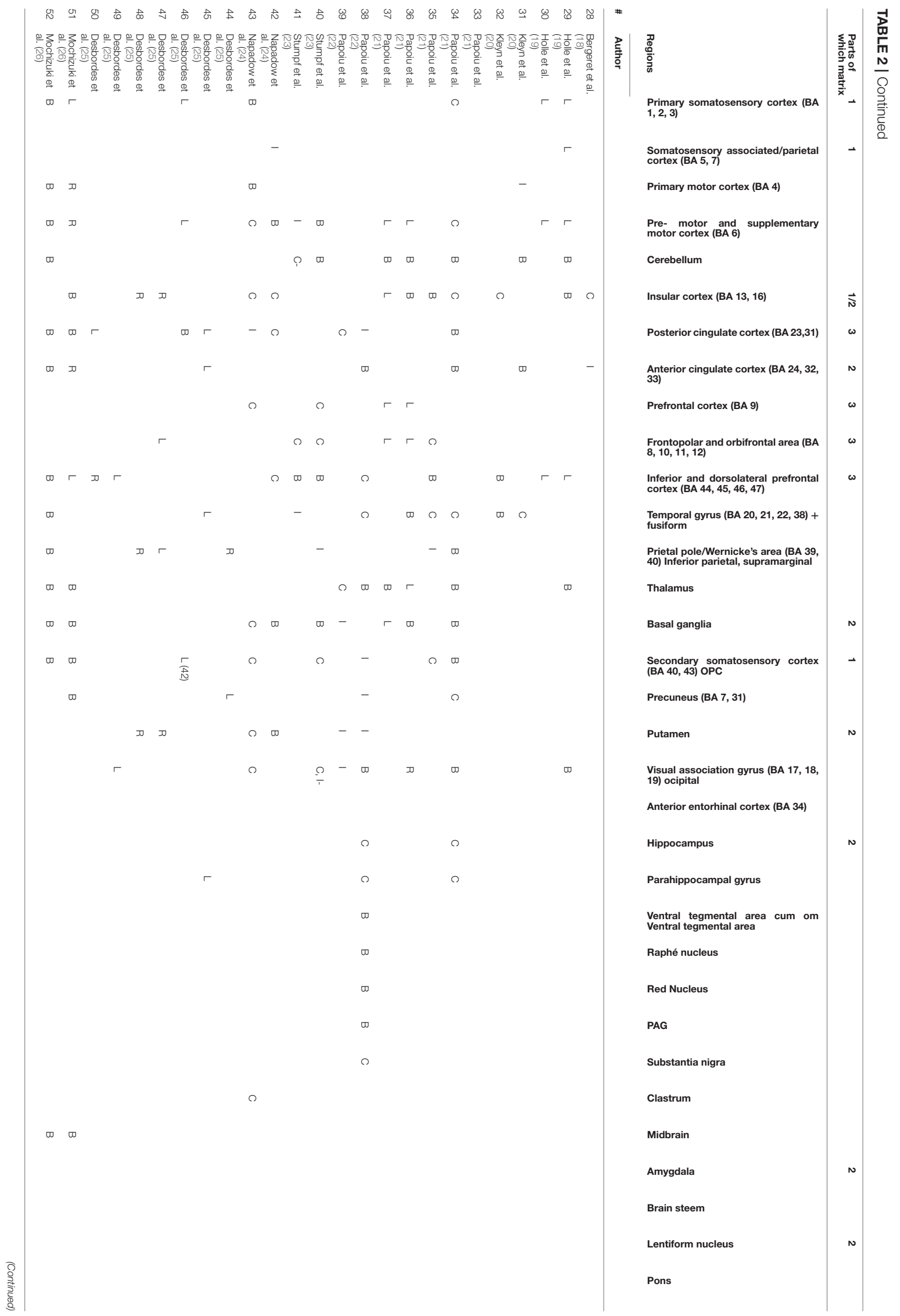


TABLE 2 | Continued Parts of
which matrix $\begin{array}{llllll}1 / 2 & 3 & 2 & 3 & 3 & 3\end{array}$

2
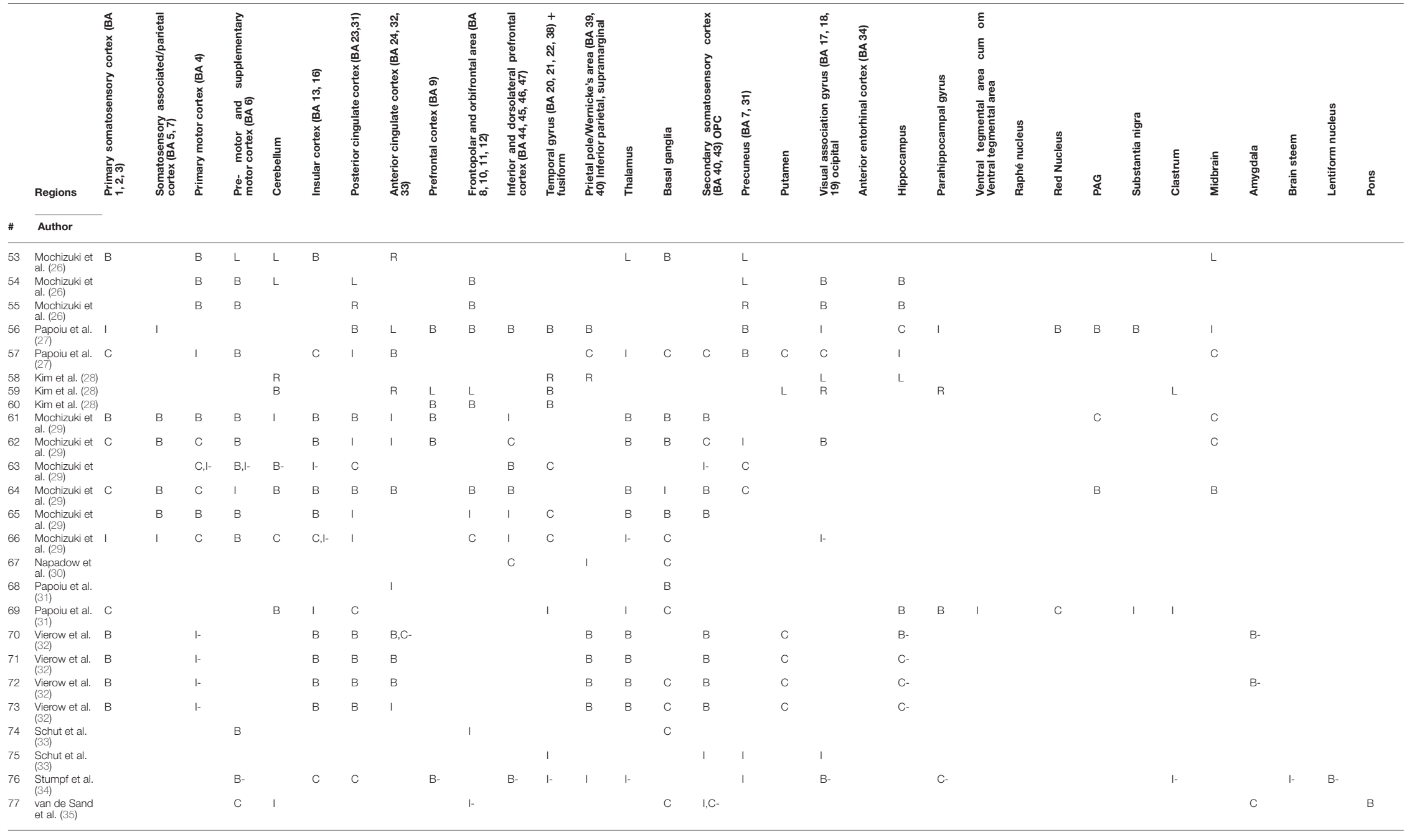


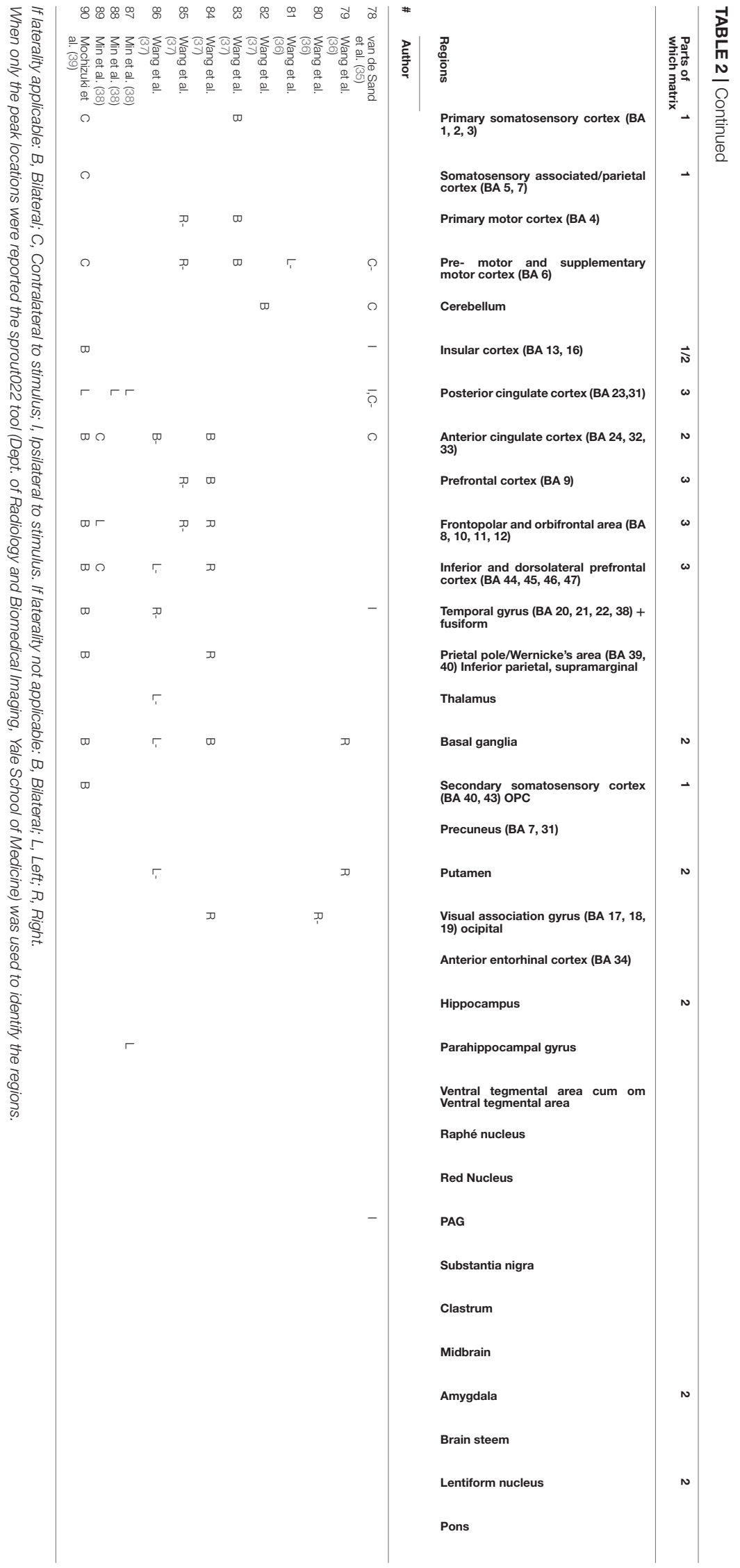



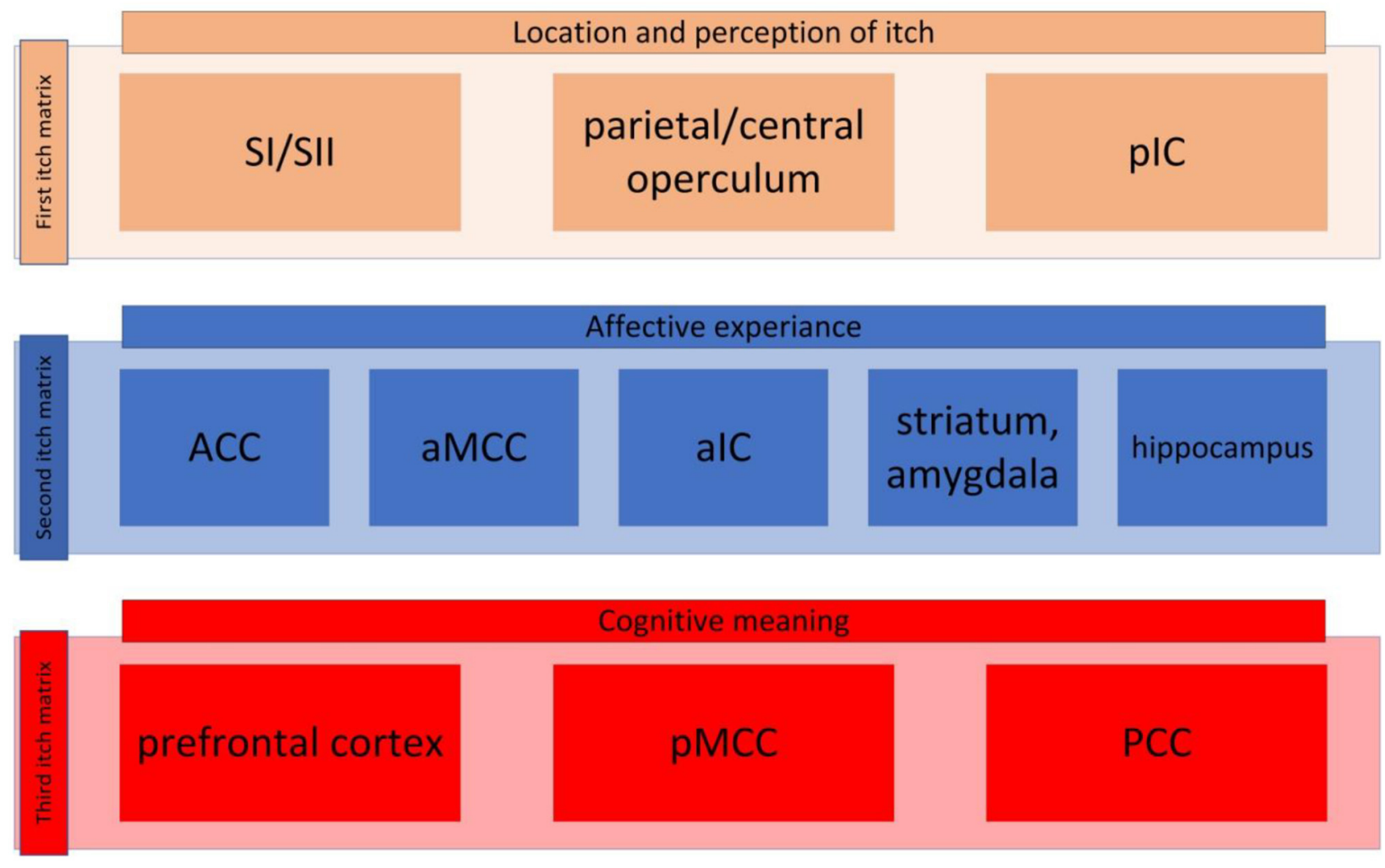

FIGURE 1 | The itch matrix categorized into three itch matrixes. First itch matrix consisted of primary and secondary sensorimotor cortex (SI and SII, respectively), the parietal/central operculum, and the posterior insular cortex (plC) (here presented in brown, this matrix is also presented in Figure 2A). The second itch matrix consisting of anterior singular cortex (ACC), anterior part of the middle cingulate cortex (aMCC), anterior part of the insular cortex (alC), amygdala, striatum and hippocampus (here presented in blue, this matrix is also presented in Figure 2B). The third matrix contains prefrontal cortex, posterior part of the middle cingulate cortex (pMCC), and posterior cingulate cortex (PCC) (here presented in red, this matrix is also presented in Figure 2C).

involved with motivation aspects of itch and/or the carving for scratching.

According to original paradigms, two other studies have reported diminished activation of these regions in tasks that change the nature of pain perception with context variations (66) or with analgesia induced by meditation (67). While the first of these shows a diminished activation in dorsal ACC and insula as the subjects switch their perception from unpleasant to pleasant (or less unpleasant) revealing the link between emotional and motivational function. The second demonstrate that experienced Zen meditators can reduce activity of their prefrontal medial cortex, amygdala and hippocampus regions at the expense of an increased activity in dorsal ACC or insula which still belong to this second matrix but are more related to mindfulness. These articles suggest that making things more conscious by bringing activities closer to the awareness matrix (with insula as a common region) putatively lead to less harmful psychological consequences. This second matrix is more robust than the first one. Many arguments in the itch literature exist and converge about its functional role.

\section{THIRD ITCH MATRIX}

The third itch matrix would include parts of the prefrontal cortex, pMCC, and PCC (Figure 2C). This network should be involved in the subjective perception of itch. The cognitive state of the mind can affect the itch sensation e.g., emotions, obsessions, religious beliefs, disgusts, expectations, and past experiences. This pattern of activation is also present in the distraction from itch caused by the Stroop task (e.g., in the DLPFC) $(14,30,34)$. The third matrix receives and integrates information from the foregoing two and triggers behavioral response.

\section{CONCLUSION}

Knowledge of itch processing in the brain is growing thanks to brain imaging $(2,68)$. A better understanding of interactions between itch matrixes would allow a better understanding 


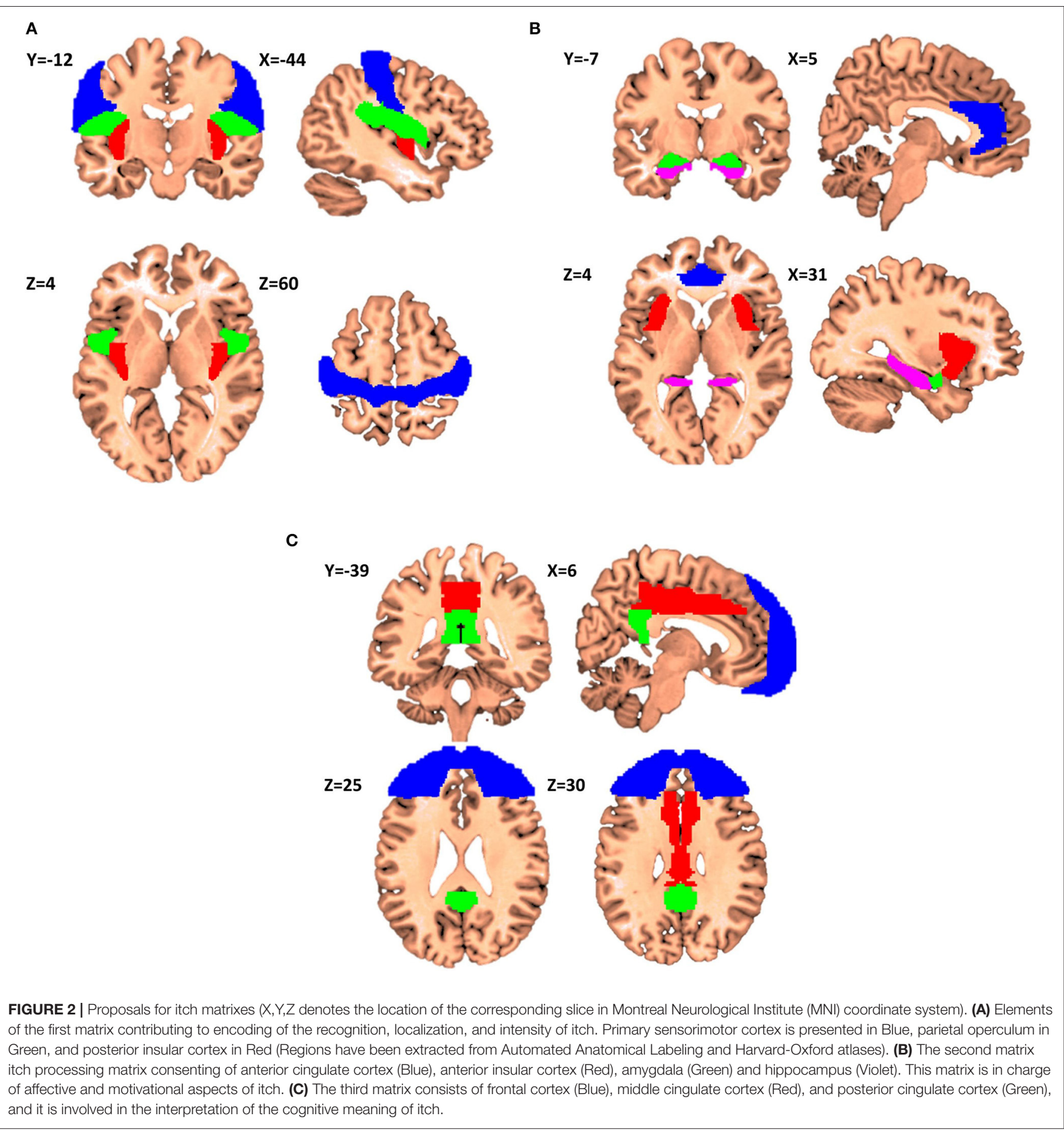

of pruritus in different cutaneous or extra-cutaneous etiologies (69).

\section{AUTHOR CONTRIBUTIONS}

LM, J-LC, DB, and OD contributed to conception and design of the study. PN organized the database and wrote the first draft of the manuscript. OD wrote sections of the manuscript. All authors contributed to manuscript revision, read, and approved the submitted version.

\section{ACKNOWLEDGMENTS}

We thank the French Society of Dermatology for providing a grant. 


\section{REFERENCES}

1. Najafi P, Carré JL, Ben Salem D, Brenaut E, Misery L, Dufor O. Central mechanisms of itch: a systematic literature review and meta-analysis. $J$ Neuroradiol. (2020) 47:450-7. doi: 10.1016/j.neurad.2019.11.005

2. Najafi P, Dufor O, Ben Salem D, Misery L, Carré JL. Itch processing in the brain. J Eur Acad Dermatol Venereol. (2020) 47:400-1. doi: 10.1111/jdv.17029

3. Hsieh JC, Hagermark O, Stahle-Backdahl M, Ericson K, Eriksson L, StoneElander S, et al. Urge to scratch represented in the human cerebral cortex during itch. J Neurophysiol. (1994) 72:3004-8. doi: 10.1152/jn.1994.72.6.3004

4. Darsow U, Drzezga A, Frisch M, Munz F, Weilke F, Bartenstein P, et al. Processing of histamine-induced itch in the human cerebral cortex: a correlation analysis with dermal reactions. $J$ Invest Dermatol. (2000) 115:1029-33. doi: 10.1046/j.1523-1747.2000.00 193.x

5. Drzezga A, Darsow U, Treede RD, Siebner H, Frisch M, Munz F, et al. Central activation by histamine-induced itch: analogies to pain processing: a correlational analysis of $\mathrm{O}-15 \mathrm{H} 2 \mathrm{O}$ positron emission tomography studies. Pain. (2001) 92:295-305. doi: 10.1016/S0304-3959(01)00271-8

6. Mochizuki H, Tashiro M, Kano M, Sakurada Y, Itoh M, Yanai K. Imaging of central itch modulation in the human brain using positron emission tomography. Pain. (2003) 105:339-46. doi: 10.1016/S0304-3959(03)0 0249-5

7. Walter B, Sadlo MN, Kupfer J, Niemeier V, Brosig B, Stark R, et al. Brain activation by histamine prick test-induced itch. J Gen Intern Med. (2005) 20:380-2. doi: 10.1111/j.0022-202X.2005.23817.x

8. Herde L, Forster C, Strupf M, Handwerker HO. Itch induced by a novel method leads to limbic deactivations - a functional MRI study. J Neurophysiol. (2007) 98:2347-56. doi: 10.1152/jn.004 75.2007

9. Leknes SG, Bantick S, Willis CM, Wilkinson JD, Wise RG, Tracey I. Itch and motivation to scratch: an investigation of the central and peripheral correlates of allergen- and histamine-induced itch in humans. J Neurophysiol. (2007) 97:415-22. doi: 10.1152/jn.00070.2006

10. Mochizuki H, Sadato N, Saito DN, Toyoda H, Tashiro M, Okamura $\mathrm{N}$, et al. Neural correlates of perceptual difference between itching and pain: A human fMRI study. Neuroimage. (2007) 36:706-17. doi: 10.1016/j.neuroimage.2007.04.003

11. Valet M, Pfab F, Sprenger T, Wöller A, Zimmer C, Behrendt H, et al. Cerebral Processing of histamine-induced itch using short-term alternating temperature modulation - an fMRI study. J Invest Dermatol. (2007) 128:42633. doi: $10.1038 /$ sj.jid. 5701002

12. Schneider G, Stander S, Burgmer M, Driesch G, Heuft G, Weckesser M. Significant differences in central imaging of histamine-induced itch between atopic dermatitis and healthy subjects. Eur J Pain. (2008) 12:834-41. doi: 10.1016/j.ejpain.2007.12.003

13. Yosipovitch G, Ishiuji Y, Patel TS, Hicks MI, Oshiro Y, Kraft RA, et al. The brain processing of scratching. J Invest Dermatol. (2008) 128:1806-11. doi: $10.1038 /$ jid.2008.3

14. Ishiuji Y, Coghill R, Patel T, Oshiro Y, Kraft R, Yosipovitch G. Distinct patterns of brain activity evoked by histamine-induced itch reveal an association with itch intensity and disease severity in atopic dermatitis. Br J Dermatol. (2009) 161:1072-80. doi: 10.1111/j.1365-2133.2009.09308.x

15. Mochizuki H, Inui K, Tanabe HC, Akiyama LF, Otsuru N, Yamashiro K, et al. Time course of activity in itch-related brain regions: a combined MEG -fMRI study. J Neurophysiol. (2009) 102:2657-66. doi: 10.1152/jn.00460.2009

16. Vierow V, Fukuoka M, Ikoma A, Dörfler A, Handwerker HO, Forster C. Cerebral representation of the relief of itch by scratching. J Neurophysiol. (2009) 102:3216-24. doi: 10.1152/jn.00207.2009

17. Pfab F, Valet M, Sprenger T, Huss-Marp J, Athanasiadis GI, Baurecht HJ, et al. Temperature modulated histamine-itch in lesional and nonlesional skin in atopic eczema - a combined psychophysical and neuroimaging study. Allergy. (2010) 65:84-94. doi: 10.1111/j.1398-9995.2009.02163.x

18. Bergeret L, Black D, Theunis J, Misery L, Chauveau N, Aubry F, et al. Validation of a model of itch induction for brain positron emission tomography studies using histamine iontophoresis. Acta Derm Venereol. (2011) 91:504-10. doi: 10.2340/00015555-1067
19. Holle H, Warne K, Seth AK, Critchley HD, Ward J. Neural basis of contagious itch and why some people are more prone to it. PNAS. (2012) 109:19816-21. doi: 10.1073/pnas.1216160109

20. Kleyn C e., McKie S, Ross A, Elliott R, Griffiths CEM. A temporal analysis of the central neural processing of itch. Br J Dermatol. (2012) 166:994-1001. doi: $10.1111 / j .1365-2133.2012 .10849 . x$

21. Papoiu AD, Coghill RC, Kraft RA, Wang H, Yosipovitch G. A tale of two itches. Common features and notable differences in brain activation evoked by cowhage and histamine induced itch. Neuroimage. (2012) 59:3611-23. doi: 10.1016/j.neuroimage.2011.10.099

22. Papoiu AD, Nattkemper LA, Sanders KM, Kraft RA, Chan YH, Coghill RC, et al. Brain's reward circuits mediate itch relief: a functional MRI study of active scratching. PLoS ONE. (2013) 8:e82389. doi: 10.1371/journal.pone.0082389

23. Stumpf A, Burgmer M, Schneider G, Heuft G, Schmelz M, Phan NQ, et al. Sex differences in itch perception and modulation by distraction an fMRI pilot study in healthy volunteers. PLoS ONE. (2013) 8:e0079123. doi: 10.1371/journal.pone. 0079123

24. Napadow V, Li A, Loggia ML, Kim J, Schalock PC, Lerner E, et al. The brain circuitry mediating antipruritic effects of acupuncture. Cereb Cortex. (2014) 24:873-82. doi: 10.1093/cercor/bhs363

25. Desbordes G, Li A, Loggia ML, Kim J, Schalock PC, Lerner E, et al. Evoked itch perception is associated with changes in functional brain connectivity. Neuroimage Clin. (2014) 7:213-21. doi: 10.1016/j.nicl.2014.12.002

26. Mochizuki H, Tanaka S, Morita T, Wasaka T, Sadato N, Kakigi R. The cerebral representation of scratching-induced pleasantness. J Neurophysiol. (2014) 111:488-98. doi: 10.1152/jn.00374.2013

27. Papoiu ADP, Emerson NM, Patel TS, Kraft RA, Valdes-Rodriguez R, Nattkemper LA, et al. Voxel-based morphometry and arterial spin labeling fMRI reveal neuropathic and neuroplastic features of brain processing of itch in end-stage renal disease. J Neurophysiol. (2014) 112:1729-38. doi: 10.1152/jn.00827.2013

28. Kim HJ, Park JB, Lee JH, Kim I-H. How stress triggers itch: a preliminary study of the mechanism of stress-induced pruritus using fMRI. Int J Dermatol. (2015) 55:434-42. doi: 10.1111/ijd.12864

29. Mochizuki H, Papoiu ADP, Nattkemper LA, Lin AC, Kraft RA, Coghill RC, et al. Scratching induces overactivity in motor-related regions and reward system in chronic itch patients. J Invest Dermatol. (2015) 135:2814-23. doi: $10.1038 /$ jid.2015.223

30. Napadow V, Li A, Loggia ML, Kim J, Mawla I, Desbordes G, et al. The imagined itch: brain circuitry supporting nocebo-induced itch in atopic dermatitis patients Allergy. (2015) 70:1485-92. doi: 10.1111/all. 12727

31. Papoiu ADP, Kraft RA, Coghill RC, Yosipovitch G. Butorphanol suppression of histamine itch is mediated by nucleus accumbens and septal nuclei: a pharmacological fMRI study. J Invest Dermatol. (2015) 135:560-8. doi: $10.1038 /$ jid.2014.398

32. Vierow V, Forster C, Vogelgsang R, Dörfler A, Handwerker H. Cerebral networks linked to itch-related sensations induced by Histamine and Capsaicin. Acta Dermato Venereologica. (2015) 95:645-52. doi: 10.2340/00015555-2006

33. Schut C, Mochizuki H, Grossman SK, Lin AC, Conklin CJ, Mohamed FB, et al. Brain processing of contagious itch in patients with atopic dermatitis. Front Psychol. (2017) 8:1267. doi: 10.3389/fpsyg.2017.01267

34. Stumpf A, Pfleiderer B, Schneider G, Heuft G, Schmelz M, Phan NQ, et al. Distraction from itch shows brainstem activation without reduction in experimental itch sensation. Acta Derm Venereol. (2017) 97:1074-80. doi: 10.2340/00015555-2732

35. van de Sand MF, Menz MM, Sprenger C, Büchel C. Nocebo-induced modulation of cerebral itch processing - an fMRI study. NeuroImage. (2018) 166:209-18. doi: 10.1016/j.neuroimage.2017.10.056

36. Wang Y, Fang J-L, Cui B, Liu J, Song P, Lang C, et al. The functional and structural alterations of the striatum in chronic spontaneous urticarial. Sci Rep. (2018) 8:1725. doi: 10.1038/s41598-018-19962-2

37. Wang Y, Fang J, Song P, Bao Y, Song W, Liu J, et al. The dysfunction of the cerebellum and its cerebellum-reward-sensorimotor loops in chronic spontaneous urticaria. Cerebellum. (2018) 17:507-16. doi: 10.1007/s12311-018-0933-6 
38. Min S, Kim K-W, Jung W-M, Lee M-J, Kim Y-K, Chae Y, et al. Acupuncture for histamine-induced itch: association with increased parasympathetic tone and connectivity of putamen-midcingulate cortex. Front Neurosci. (2019) 13:215. doi: 10.3389/fnins.2019.00215

39. Mochizuki H, Hernandez LE, Yosipovitch G, Sadato N, Kakigi R. The functional network processing acute electrical itch stimuli in humans. Front Physiol. (2019) 10:555. doi: 10.3389/fphys.2019.00555

40. Fenton BW, Shih E, Zolton J. The neurobiology of pain perception in normal and persistent pain. Pain Manage. (2015) 5:297-317. doi: 10.2217/pmt.15.27

41. Xiang Y, Wang Y, Gao S, Zhang X, Cui R. Neural mechanisms with respect to different paradigms and relevant regulatory factors in empathy for pain. Front Neurosci. (2018) 12:507. doi: 10.3389/fnins.2018.00507

42. Apkarian AV, Bushnell MC, Treede RD, Zubieta JK. Human brain mechanisms of pain perception and regulation in health and disease. Eur J Pain. (2005) 9:463-84. doi: 10.1016/j.ejpain.2004.11.001

43. Dong WK, Salonen LD, Kawakami Y, Shiwaku T, Kaukoranta EM, Martin RF. Nociceptive responses of trigeminal neurons in SII-7b cortex of awake monkeys. Brain Res. (1989) 484:314-24. doi: 10.1016/0006-8993(89)90375-2

44. Dong WK, Chudler EH, Sugiyama K, Roberts VJ, Hayashi T. Somatosensory, multisensory, and task-related neurons in cortical area $7 \mathrm{~b}$ (PF) of unanesthetized monkeys. J Neurophysiol. (1994) 72:542-64. doi: 10.1152/jn.1994.72.2.542

45. Timmermann L, Ploner M, Haucke K, Schmitz F, Baltissen R, Schnitzler A. Differential coding of pain intensity in the human primary and secondary somatosensory cortex. J Neurophysiol. (2001) 86:1499-503. doi: 10.1152/jn.2001.86.3.1499

46. Bornhövd K, Quante M, Glauche V, Bromm B, Weiller C, Büchel C. Painful stimuli evoke different stimulus-response functions in the amygdala, prefrontal, insula and somatosensory cortex: a singletrial fMRI study. Brain. (2002) 125:1226-36. doi: 10.1093/brain/awf137

47. Frot M, Magnin M, Mauguière F, Garcia-Larrea L. Human SII and posterior insula differently encode thermal laser stimuli. Cereb Cortex. (2007) 17:61020. doi: 10.1093/cercor/bhk007

48. Lee JS, Han JS, Lee K, Bang J, Lee $H$. The peripheral and central mechanisms underlying itch. BMB Rep. (2016) 49:474-96. doi: 10.5483/BMBRep.2016.49.9.108

49. Roberts CA, Stancak A, Fallon N, Thomas A, Kirkham TC. Where is itch represented in the brain, and how does it differ from pain? An activation likelihood estimation meta-analysis of experimentally-induced itch. J Invest Dermatol. (2019) 139:2245-8. doi: 10.1016/j.jid.2019.04.007

50. Craig AD. The sentient self. Brain Struct Funct. (2010) 214:563-77. doi: 10.1007/s00429-010-0248-y

51. Craig AD. Significance of the insula for the evolution of human awareness of feelings from the body. Ann N Y Acad Sci. (2011) 1225:72-82. doi: 10.1111/j.1749-6632.2011.05990.x

52. Mochizuki H, Kakigi R. Central mechanisms of itch Clin Neurophysiol. (2015) 126:1650-60. doi: 10.1016/j.clinph.2014.11.019

53. Mochizuki H, Yosipovitch G. Neuroimaging of itch as a tool of assessment of chronic itch and its management. Handb Exp Pharmacol. (2015) 226:57-70. doi: 10.1007/978-3-662-44605-8_4

54. Kurth F, Eickhoff SB, Schleicher A, Hoemke L, Zilles K, Amunts K. Cytoarchitecture and probabilistic maps of the human posterior insular cortex. Cereb Cortex. (2010) 20:1448-61. doi: 10.1093/cercor/bhp208

55. Mazzola L, Isnard J, Peyron R, Guenot M, Mauguiere F. Somatotopic organization of pain responses to direct electrical stimulation of the human insular cortex. Pain. (2009) 146:99-104. doi: 10.1016/j.pain.2009.07.014
56. Iadarola MJ, Berman KF, Zeffiro TA, Byas-Smith MG, Gracely RH, Max MB, et al. Neural activation during acute capsaicin-evoked pain and allodynia assessed with PET. Brain. (1998) 121:931-47. doi: 10.1093/brain/121.5.931

57. Peyron R, Schneider F, Faillenot I, Convers P, Barral FG, GarciaLarrea L, et al. An fMRI study of cortical representation of mechanical allodynia in patients with neuropathic pain. Neurology. (2004) 63:1838-46. doi: 10.1212/01.WNL.0000144177.61125.85

58. Schweinhardt P, Glynn C, Brooks J, Mcquay H, Jack T, Chessell I, et al. An fMRI study of cerebral processing of brush-evoked allodynia in neuropathic pain patients. Neuroimage. (2006) 32:256-65. doi: 10.1016/j.neuroimage.2006.03.024

59. Seifert F, Maihöfner C. Representation of cold allodynia in the human brain-a functional MRI study. Neuroimage. (2007) 35:1168-80. doi: 10.1016/j.neuroimage.2007.01.021

60. Ducreux D, Attal N, Parker F, Bouhassira D. Mechanisms of central neuropathic pain: a combined psychophysical and fMRI study in syringomyelia. Brain. (2006) 129:963-76. doi: 10.1093/brain/awl016

61. Geuter S, Boll S, Eippert F, Büchel C. Functional dissociation of stimulus intensity encoding and predictive coding of pain in the insula. eLife. (2017) 6:e24770. doi: 10.7554/eLife.24770.016

62. Vogt BA. Pain and emotion interactions in subregions of the cingulate gyrus. Nat Neurosci. (2005) 6:533-44. doi: 10.1038/nrn1704

63. Vogt BA. Midcingulate cortex: Structure, connections, homologies, functions and diseases. J Chem Neuroanat. (2016) 74:28-46. doi: 10.1016/j.jchemneu.2016.01.010

64. Gu X, Hof PR, Friston KJ, Fan J. Anterior insular cortex and emotional awareness. J Comp Neurol. (2013) 521:3371-88. doi: 10.1002/cne.23368

65. Sanders KM, Akiyama T. The vicious cycle of itch and anxiety. Neurosci Biobehav Rev. (2018) 87:17-26. doi: 10.1016/j.neubiorev.2018. 01.009

66. Leknes S, Berna C, Lee MC, Snyder GD, Biele G, Tracey I. The importance of context: when relative relief renders pain pleasant. Pain. (2013) 154:402-10. doi: 10.1016/j.pain.2012.11.018

67. Grant J, Courtemanche J, Rainville P. A non-elaborative mental stance and decoupling of executive and pain-related cortices predicts low pain sensitivity in Zen meditators. Pain. (2011) 152:150-6. doi: 10.1016/j.pain.2010. 10.006

68. Cheddad El Aouni M, Ben Salem D, Misery L. Functional MRI of pruritus. J Neuroradiol. (2020) 47:400-1. doi: 10.1016/j.neurad.2020. 09.001

69. Najafi P, Ben Salem D, Carré JL, Misery L, Dufor O. Functional and anatomical brain connectivity in psoriasis patients and healthy controls: a pilot brain imaging study after exposure to mentally induced itch. J Eur Acad Dermatol Venereol. (2020) 34:2557-65. doi: 10.1111/jdv.16441

Conflict of Interest: The authors declare that the research was conducted in the absence of any commercial or financial relationships that could be construed as a potential conflict of interest.

Copyright (C) 2021 Najafi, Misery, Carré, Ben Salem and Dufor. This is an open-access article distributed under the terms of the Creative Commons Attribution License (CC $B Y)$. The use, distribution or reproduction in other forums is permitted, provided the original author(s) and the copyright owner(s) are credited and that the original publication in this journal is cited, in accordance with accepted academic practice. No use, distribution or reproduction is permitted which does not comply with these terms. 